\title{
Sliding mode control and Lyapunov based guidance law with impact time constraints
}

\author{
Xiaojian Zhang, Mingyong Liu*, and Yang Li \\ School of Marine Science and Technology, Northwestern Polytechnical University, Xi' an 710021, China
}

\begin{abstract}
This paper analyses the issue of impact time control of super-cavitation weapons impact fixed targets which mainly refer to the ships or submarines who lost power, but still have combat capability. Control over impact time constraints of guidance law (ITCG) is derived by using sliding mode control (SMC) and Lyapunov stability theorem. The expected impact time is realized by using the notion of attack process and estimated time-to-go to design sliding mode surface (SMS). ITCG contains equivalent and discontinuous guidance laws, once state variables arrive at SMS, the equivalent guidance law keeps the state variables on SMS, then the discontinuous guidance law enforces state variables to move and reach SMS. The singularity problem of ITCG is also analyzed. Theoretical analysis and numerical simulation results are given to test the effectiveness of ITCG designed in this paper.
\end{abstract}

Keywords: Lyapunov stability, sliding mode control, impact time, salvo attack, time-to-go, guidance law.

DOI: $10.21629 / J S E E .2017 .06 .16$

\section{Introduction}

The purpose of terminal navigation is to guide an interceptor to a desired location. Proportional navigation guidance (PNG) has been widely used in the guided weapons, and its main control strategy is to generate an instruction in proportion to the line-of-sight (LOS) angle rate [1]. However, recently guided weapons have been equipped with defense systems, for improving the attack performance. For example, missiles, high speed antiships or antisubmarine supercavitation torpedoes have been developed, as a salvo attack where several high speed super-cavitation torpedoes hit the target simultaneously is one of the ways to destroy some large targets [2].

Although there are many published literatures studying impact angle control over guidance law (IACG) [3-9], only few papers which studied the impact time constraints

\footnotetext{
Manuscript received September 03, 2016.

*Corresponding author.

This work was supported by the National Natural Science Foundation of China (51379176; 51679201).
}

of guidance (ITCG) or guidance law with impact time and impact angle (IT \& IACG) are available in open literatures.

However, guidance laws have been developed using sliding mode control (SMC), optimal control, linear matrix inequality and so on to ameliorate traditional guidance capability or realize some certain purpose [10]. For example, ITCG of the interceptor is necessary to realize successful salvo attack against arbitrarily non-maneuvering targets [11-14]. Therefore, like IACG, ITCG can also enhance guidance capability.

Under the basis of PNG, combining a term of impact time error, Zhang et al. designed an ITCG with field-ofview constraints for intercepting stationary targets [15]. Zhang et al. presented an IACG under the biased-PNG (BPNG) [16], then designed a control law which enforces impact time error to go to zero before final collision. Jeon et al. presented an ITCG which bases on a linearized approximation of the engagement dynamics for stationary targets [10]. The ITCG consists a PNG and feedback control which enforces the error of impact time to converge to zero. Different from [10], an ITCG was proposed, containing feedback control which reduced the miss distance and feedforward control which adjusted the impact time [11].

Because SMC has a good robust, in [8,17], a guidance law was derived against a fixed target, then extended against non-maneuvering targets based on sliding mode surface (SMS) which is designed based on the concept of the collision course and the time-to-go. In [18], Zhao et al. firstly designed a guidance law under the nonlinear kinematics using the time varying SMS which was constructed by two feedback controllers to satisfy the requirement of impact time and impact angle. In [19], Saleem and Ratnoo proposed a guidance law with the heading angle variation and feedback form solution to achieve impact time against stationary targets. In [20], Kumar and Ghose proposed a guidance strategy based on switching between ITCG and IACG. 
Motivated by [8], Cho et al. [21] proposed an IACG based on SMC against a stationary target and extended it to constant velocity targets as similarly as in [17]. However, Cho et al. proposed a guidance law based on SMC using a positive continuous nonlinear function of the missile's leading angle which not only satisfies the impact time requirement, but also avoids the singularity problem of the guidance command. In [22], IT and IACG were proposed against stationary targets by using a novel second order SMC (SOSMC) based on the backstepping concept. Similarly, Zhao et al. [23] proposed the IT and IACG using the same method, but from different points of views. Zhou et al. [24] presented a different IACG by using SOSMC theory and finite time converge theory, which can realize expected impact angle. In [25], for intercepting stationary targets, Kim and Lee proposed IT and IACG based on a polynomial function with coefficients which were designed for realizing impact angle and impact time.

Based on the concept of time-to-go, Cho and Kim [26] investigated the time-to-go of the pure proportional navigation and considered nonlinear engagement kinematics, then proposed ITCG for intercepting stationary targets. In [27], considering the impact time constraints, Lee proposed ITCG using linear quadratic optimal theory and time-to-go weighted to minimize the control energy. Similar to [27], Ryoo et al. [28] presented an ITCG based on solution of time-to-go by using linear quadratic optimal. Different from [26-28], Snyder and Hull developed an ITCG based on explicit of time-to-go which was deduced using linear dynamic [29].

Besides the above literatures, modern control theories like the famous Lyapunov stability theorem have developed the new area of guidance laws [2,30-33].

In [2], Kim et al. proposed an ITCG based on Lyapunov stability theorem and SMC, then analyzed stability and singularity of the ITCG command which has a simple form to be implemented easily. In [30], an ITCG was presented based on Lyapunov stability theorem through adopting the notion of nonzero effort miss. Yanushevsky et al. [31] presented an ITCG which contained two parts, the traditional PNG and an additional term using the concept of Lyapunov stability theorem. In [32], a new ITCG based on Lyapunov stability theorem like in [31] was presented, in addition, a parameter optimization method was also presented to adjust flight trajectory to satisfy IACG.

For intercepting stationary targets, we propose a nonlinear ITCG which is motivated by [2]. However, unlike the approach in [2], the guidance law commands contain two terms: the equivalent guidance law and the discontinuous guidance law, in which the equivalent guidance law keeps the state variables in the SMS, and the other drives state variables towards the SMS.

The remainder of the paper is addressed as follows. In Section 2, a two dimensional plane of the kinematics and equation of motion are stated. In Section 3, the ITCG is designed based on SMC and Lyapunov stability theorem, and the satiability of the system is also discussed. Section 4 presents the numerical simulation, and simulation results are illustrated to test ITCG designed in this paper comparing with [2]. Finally, the conclusion is drawn.

\section{Problem formulation}

A two dimensional plane of kinematic geometrical relation is discussed to design guidance laws as in coordinate system $X O Z$ in Fig. 1. The interceptor is considered as a point mass, and the target is assumed as stationary. In this study, it is assumed that the interceptor is moving at a constant speed $v_{m}$. The lateral acceleration $a_{m}$ of the interceptor is assumed to be perpendicular to the velocity direction $v_{m}$.

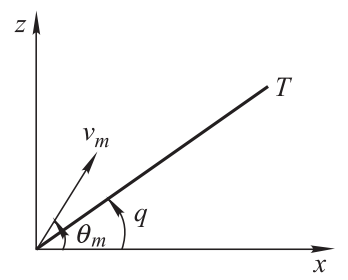

Fig. 1 Two dimensional kinematic geometry diagram

On the basis of the principles of kinematics, the kinematic equation of the guidance system is written as follows:

$$
\begin{gathered}
\dot{r}=-v_{m} \cos \left(q-\theta_{m}\right) \\
r \dot{q}=v_{m} \sin \left(q-\theta_{m}\right) \\
\dot{\theta}_{m}=a_{m} / v_{m}=a_{c}
\end{gathered}
$$

where $r$ denotes the relative range and $\dot{r}$ denotes the relative velocity between the interceptor and the target, $q$ denotes the LOS angle and $\dot{q}$ denotes the LOS angle rate between the interceptor and the target, $v_{m}$ is the velocity of the interceptor, and $\theta_{m}$ is the heading angle.

The purpose of this paper is to design a guidance law which guarantees the interceptor hits the target not only with zero miss distance, but also with an expected impact time. How to achieve the guidance purposes is illustrated in the following sections.

\subsection{Switching surface design}

In the engagement plane, the impact time can be expressed as

$$
t_{f}=t+t_{g o}
$$

where $t_{f}$ means the final impact time of the interceptor to the target, $t$ means the elapsed time after launching an interceptor, and the $t_{g o}$ denotes the time to go. The accurate 
$t_{g o}$ estimation method is essential, in other words, the impact time guidance performance strongly depends on the $t_{g o}$ estimation way. As the flight path is curved generally when the interceptor is homing to the target, the conventional $t_{g o}$ estimation method as $t_{g o}=-r / \dot{r}$ may generate great error which may reduce guidance performance. In this paper, we use the improved $t_{g o}$ estimation method for intercepting stationary targets represented in [2] as follows:

$$
t_{g o}=\frac{r}{v_{m}}\left(1+\frac{\left(q-\theta_{m}\right)^{2}}{10}\right) .
$$

Define SMS as

$$
s=t_{f}-t_{d}
$$

where $t_{d}$ is the desired impact time, and $t_{d}$ should be set by considering the given interceptors, simultaneous impact, i.e. $t_{d} \geqslant t_{f \max }, t_{f \max }=\max \left[t_{f 1}, t_{f 2}, t_{f 4}, \ldots, t_{f n}\right]$, $t_{f i}(i=1,2,3, \ldots, n)$ is one of the interceptors impact time estimated by PNG.

In order to ensure the existence of sliding mode, the following assumptions should be satisfied.

Assumption 1 The SMS should be differentiable.

Assumption 2

$$
\frac{1}{2} \frac{\mathrm{d}}{\mathrm{d} t} s^{2}<-\eta|s|
$$

where $\eta$ is a constant, and $\eta>0$.

From the above analysis, the SMS (6) should be kept zero neighborhood, before and when the interceptor hits the target. Hence, ITCG designed in this paper should guarantee state variables to reach and move along SMS, so that expected impact time can be realized when miss distance goes to zero.

\subsection{SMC and Lyapunov based SL-ITCG design}

The ITCG which will enforce the SMS $s=0$ is designed as

$$
a_{c}=a_{m}^{e q}+a_{m}^{d i s} .
$$

For designing ITCG $a_{c}$, we define the Lyapunov function as

$$
V=s^{2} / 2 \text {. }
$$

Differentiating (9), $\dot{V}$ can be obtained as

$$
\dot{V}=s \dot{s}=s\left(1+\dot{t}_{g o}\right)
$$

The time derivative of (5) can be obtained as

$$
\begin{gathered}
\dot{t}_{g o}=\left\{1+\frac{\left(q-\theta_{m}\right)^{2}}{10}\right\} \frac{\dot{r}}{v_{m}}+\frac{r\left(q-\theta_{m}\right)}{5 v_{m}}\left(\dot{q}-\dot{\theta}_{m}\right)= \\
\left\{1+\frac{\left(q-\theta_{m}\right)^{2}}{10}\right\} \frac{\dot{r}}{v_{m}}+\frac{r\left(q-\theta_{m}\right)}{5 v_{m}}\left(\dot{q}-a_{c}\right) .
\end{gathered}
$$

Substituting (11) into (10), we can get

$$
\begin{gathered}
\dot{V}=s \dot{s}= \\
s\left[1+\left\{1+\frac{\left(q-\theta_{m}\right)^{2}}{10}\right\} \frac{\dot{r}}{v_{m}}+\frac{r\left(q-\theta_{m}\right)}{5 v_{m}}\left(\dot{q}-a_{c}\right)\right] .
\end{gathered}
$$

Using the kinematic equations (1)-(3) and (8), the equivalent ITCG $a_{m}^{e q}$ can be got by making $\dot{V} \leqslant 0$, and from (12), the equivalent guidance law is given as

$$
\begin{aligned}
a_{m}^{e q}= & \dot{q}+\frac{5 v_{m}\left\{1-\cos \left(q-\theta_{m}\right)\right\}}{r\left(q-\theta_{m}\right)}- \\
& \frac{v_{m}\left(q-\theta_{m}\right)}{2 r} \cos \left(q-\theta_{m}\right) .
\end{aligned}
$$

Once the state variables arrive at SMS, $a_{m}^{e q}$ will keep the guidance state variables in that SMS. Simultaneously the discontinuous ITCG $a_{m}^{d i s}$ enforces the guidance state variables toward and arrive at SMS, then moves along that mode. Here, we design the discontinuous guidance law as

$$
a_{m}^{d i s}=k \operatorname{sign}(s)
$$

where $k$ is the constant coefficient of the discontinuous ITCG $a_{m}^{d i s}$ and $k>0$.

To obtain the bound of constant coefficient of $k$, under the above assumptions, we can use the Lyapunov function approach and combine (9), (12), (13) and (14) and get

$$
\dot{V}=-k s\left(r\left(q-\theta_{m}\right) / 5 v_{m}\right) \operatorname{sign}(s) .
$$

Now, from (15), if we choose $k$ as

$$
k=5 m v_{m} /\left(q-\theta_{m}\right) r
$$

where $m>0, \dot{s}$ can be written as

$$
\dot{s}=-m \operatorname{sign}(s) .
$$

Equation (15) decreases to

$$
\dot{V}=-k \operatorname{sign}(s) r\left(q-\theta_{m}\right) / 5 v_{m}=-m|s|
$$

which is negative definite.

Under the condition of (18), state variables of the guidance system can be guaranteed to reach the SMS, then interceptor can attack target with the desired impact time. Therefore, ITCG can be rewritten as

$$
\begin{aligned}
& a_{c}=a_{m}^{e q}+a_{m}^{d i s}=\dot{q}+\frac{5 v_{m}\left\{1-\cos \left(q-\theta_{m}\right)\right\}}{r\left(q-\theta_{m}\right)}- \\
& \frac{v_{m}\left(q-\theta_{m}\right)}{2 r} \cos \left(q-\theta_{m}\right)+\frac{5 m v_{m}}{r\left(q-\theta_{m}\right)} \operatorname{sign}(s) .
\end{aligned}
$$

Note that, from (18), we know that $\dot{V}$ is negative definite, and the condition for the Lyapunov stability is satisfied. 
From (19), if $q-\theta_{m}=0$, we can get that two terms may be singularity problems as follows:

$$
\begin{gathered}
l=\frac{5 v_{m}\left\{1-\cos \left(q-\theta_{m}\right)\right\}}{r\left(q-\theta_{m}\right)} \\
f=\frac{5 m v_{m}}{r\left(q-\theta_{m}\right)} \operatorname{sign}(s) .
\end{gathered}
$$

Remark Technically, $r \neq 0$ when the interception happens, and $r \in[0.1,0.25] \mathrm{m}$. That is due to the size of the interceptor [34].

As $q-\theta_{m}$ goes to zero, using the L'Hopital's rule, the limit form of the first term $l$ sees

$$
\begin{gathered}
\lim _{q-\theta_{m} \rightarrow 0} \frac{5 v_{m}\left\{1-\cos \left(q-\theta_{m}\right)\right\}}{r\left(q-\theta_{m}\right)}= \\
\frac{5 v_{m}}{r} \lim _{q-\theta_{m} \rightarrow 0} \frac{\sin \left(q-\theta_{m}\right)}{\dot{q}-\dot{\theta}_{m}}\left(\dot{q}-\dot{\theta}_{m}\right)= \\
\frac{5 v_{m}}{r} \lim _{q-\theta_{m} \rightarrow 0} \sin \left(q-\theta_{m}\right)=0 .
\end{gathered}
$$

Next, aiming at the second term of singularity, i.e. $a_{m}^{d i s}$, we consider the singularity conditions divided by two cases as follows:

Case 1: $q-\theta_{m}=0$ and $s=0$;

Case 2: $q-\theta_{m}=0$ and $s \neq 0$.

In Case 1, the guidance law $a_{m}^{\text {dis }}$ forms 0/0. Like (22), using the L'Hopital's rule in $a_{m}^{d i s}$ gives

$$
\lim _{q-\theta_{m} \rightarrow 0} \frac{5 m v_{m}}{r} \frac{\operatorname{sign}(s)}{q-\theta_{m}}=\frac{5 m v_{m}}{r} \lim _{q-\theta_{m} \rightarrow 0} \frac{\dot{s}}{\dot{q}-\dot{\theta}_{m}} .
$$

From (1)-(3), (8), (13), and the condition $q-\theta_{m}=0$, we can get the following results:

$$
\dot{q}=0, \quad a_{m}^{e q}=0, \quad \theta_{m}=\frac{a_{m}^{d i s}}{v_{m}}=-\frac{5 m}{r} \frac{\operatorname{sign}(s)}{q-\theta_{m}} .
$$

Substituting (17) and (24) into (23), we can get

$$
\lim _{q-\theta_{m} \rightarrow 0} \frac{5 m v_{m}}{r} \frac{\operatorname{sign}(s)}{q-\theta_{m}}=\lim _{q-\theta_{m} \rightarrow 0} \frac{m r}{5}\left(q-\theta_{m}\right)=0 .
$$

From the above analysis, it implies that the guidance command does not have the singularity problem in Case 1 .

In Case 2: $q-\theta_{m}=0$ and $s \neq 0$.

The discontinuous guidance $a_{m}^{\text {dis }}$ makes guidance laws command singularity. To avoid this singularity problem, modify the discontinuous guidance $a_{m}^{d i s}$ as follows:

$$
a_{m}^{d i s}=\frac{5 v_{m} m \operatorname{sign}(s) h\left(\gamma_{m}\right)}{r \gamma_{m}}
$$

where $\gamma_{m}=q-\theta_{m}$, and the function $h\left(\gamma_{m}\right)$ is defined as

$$
h\left(\gamma_{m}\right)=\left\{\begin{array}{l}
\operatorname{sign}\left(\gamma_{m}\right)\left(\gamma_{m}\right), \quad\left|\gamma_{m}\right|<\varepsilon_{1} \\
\frac{1-\varepsilon_{1}}{\varepsilon_{2}-\varepsilon_{1}}\left|\gamma_{m}\right|+\frac{\varepsilon_{1}\left(\varepsilon_{2}-1\right)}{\varepsilon_{2}-\varepsilon_{1}}, \quad \varepsilon_{1} \leqslant\left|\gamma_{m}\right| \leqslant \varepsilon_{2} \\
1, \quad \text { otherwise }
\end{array}\right.
$$

where $\varepsilon_{1}$ and $\varepsilon_{2}$ are small positive constants. From the function $h\left(\gamma_{m}\right)$, in the singular region the modified control component becomes $a_{m}^{\text {dis }}=5 v_{m} m \operatorname{sign}\left(\gamma_{m}\right) \operatorname{sign}(s) / r$, and it is not singularity.

\subsection{Chattering elimination}

The ITCG we propose has a good robustness and can realize fast convergence. However, there is a signum function in the discontinuous guidance command, which indicates that guidance command may switch. In fact, the response hysteresis of switch will produce system chattering. The chattering produced by the signum function can be smoothed by using a function $\operatorname{sgn}_{\delta}(x)$.

$$
\operatorname{sgn}_{\delta}(x)=2\left(\frac{1}{1+\exp (-\delta x)}-\frac{1}{2}\right)
$$

where $\delta>0$.

\section{Numerical simulations}

In order to test the performance of ITCG we propose, some numerical simulation results are listed in two scenarios.

\subsection{Different impact time}

We assume that the high speed super-cavitation torpedo's initial location is $\left(x_{0}, z_{0}\right)=(0,0) \mathrm{m}$, with initial heading angle $-\pi / 6$, the torpedo has a constant velocity $v_{m}=$ $50 \mathrm{~m} / \mathrm{s}$, and the fixed target's location is $(1000,100) \mathrm{m}$. Under the ITCG (19) we propose with parameters taken as $m=20, \delta=40$, three different expected impact time are set as $t_{d}=25 \mathrm{~s}, t_{d}=30 \mathrm{~s}, t_{d}=35 \mathrm{~s}$.

Fig. 2 -Fig. 6 show the simulation results of the LOS angle rate, trajectory, guidance command, the range between the torpedo and the target and $q-\theta_{m}$, respectively, with different expected impact time.

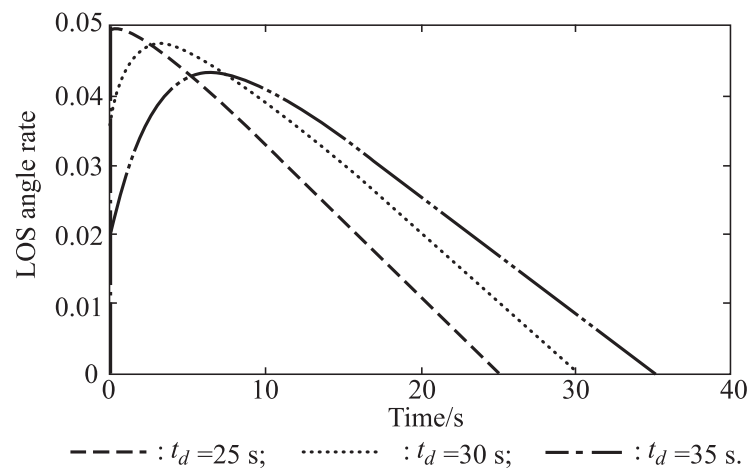

Fig. 2 LOS angle rate for different expected impact time 


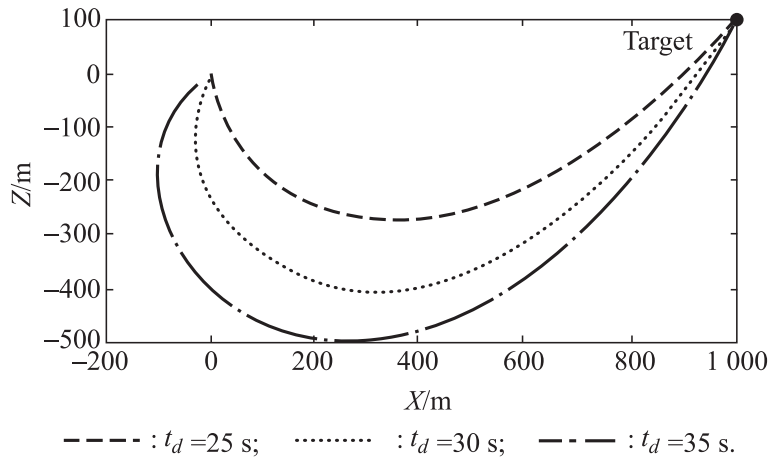

Fig. 3 Trajectories for different expected impact time

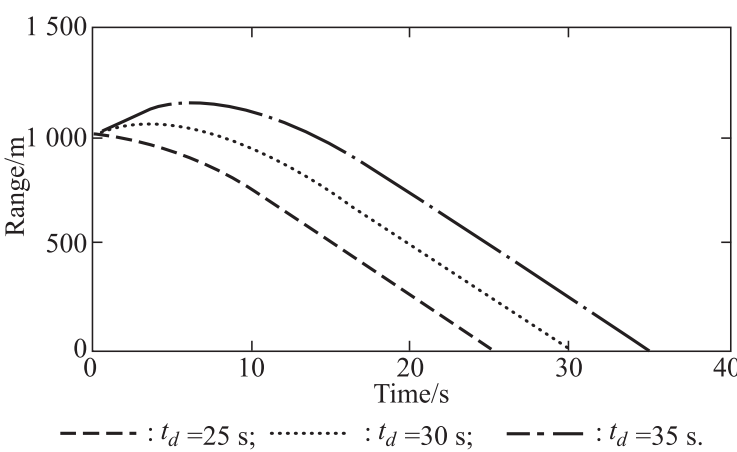

Fig. 4 Ranges with different impact time

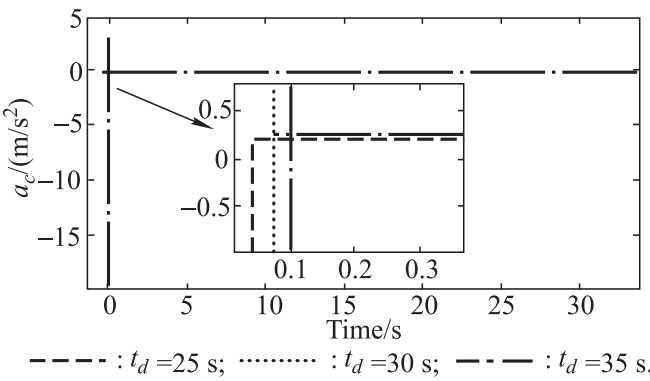

Fig. 5 Guidance commands curve

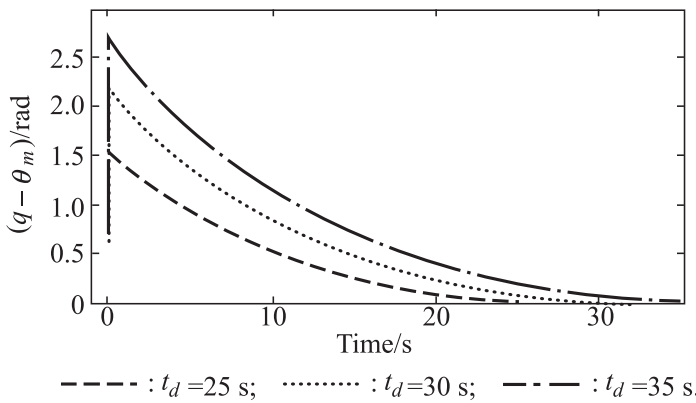

Fig. $6 q-\theta_{m}$ for different impact time

Fig. 2 shows that the LOS angle rate goes to zero when the torpedo hits the target finally, which meets the principle of parallel approach of the guidance laws.

As shown in Fig. 3 and Fig. 4, to realize the expected impact time, the torpedo flies away from the target at the beginning. In Fig. 5, the acceleration command converges to zero neighborhood and finally goes to zero. The LOS angle rate goes to zero at the final impact time given as $25 \mathrm{~s}$, $30 \mathrm{~s}$ and $35 \mathrm{~s}$, respectively. In Fig. 5, we can see that acceleration commands do not suffer from the singularity problem, when $q-\theta_{m}$ goes to zero as in Fig. 6 .

As shown in Fig. 7 and Fig. 8, the proposed ITCG has a faster convergence rate than the guidance law in [2] as well as the SMS. That is because the discontinuous acceleration command makes the system toward and move along the SMS.

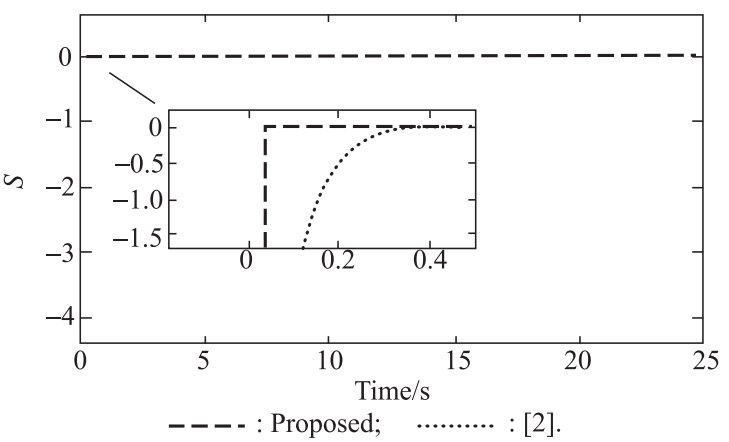

Fig. 7 Sliding mode surface curve

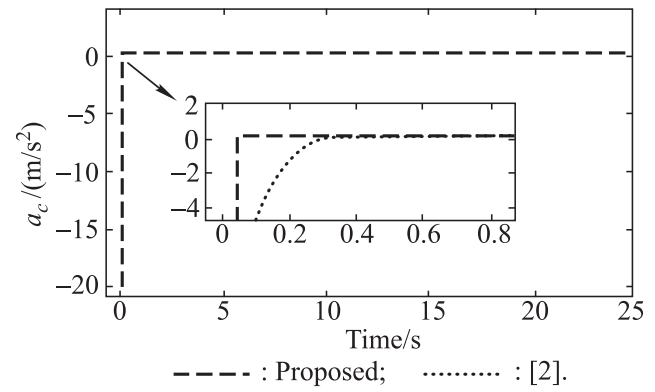

Fig. 8 Guidance law proposed and in [2] contrast with the same parameters

\subsection{Salvo attack}

We assume that three torpedoes' initial locations are $\left(x_{1}, z_{1}\right)=(0,0) \mathrm{m},\left(x_{2}, z_{2}\right)=(1000,-1000) \mathrm{m}$ and $\left(x_{3}, z_{3}\right)=(2200,100) \mathrm{m}$ and the initial heading angles are $-\pi / 6,2 \pi / 3$ and $\pi / 2$, respectively, the three torpedoes have the same constant velocity $v_{m}=50 \mathrm{~m} / \mathrm{s}$ to hit the fixed target whose location is $(1000,100) \mathrm{m}$, under the ITCG (19) with parameters taken as $m=20, \delta=40$ and $t_{d}=25 \mathrm{~s}$.

Based on the principle of the parallel approach, as in Fig. 9, the LOS angle rate converges to zero at the final desired impact time $25 \mathrm{~s}$. In Fig. 10, when $q-\theta_{m}$ converges to zero, the acceleration command has no singularity problem in Fig. 11. Fig. 12 shows different trajectories of the three torpedoes guided by ITCG proposed in this paper with the same impact time. Fig. 13 shows the range between the torpedoes and the target. 


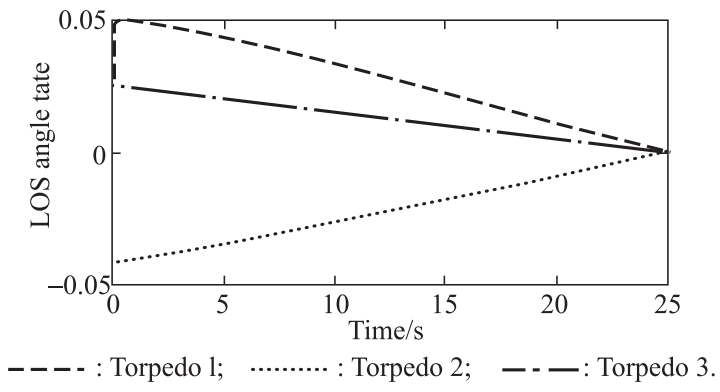

Fig. 9 LOS angle rate for different torpedoes with same impact time

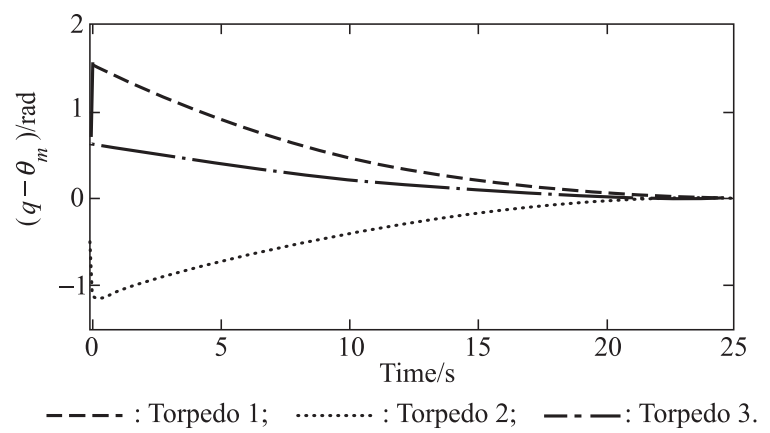

Fig. $10 q-\theta_{m}$ curve for different torpedoes with same impact time

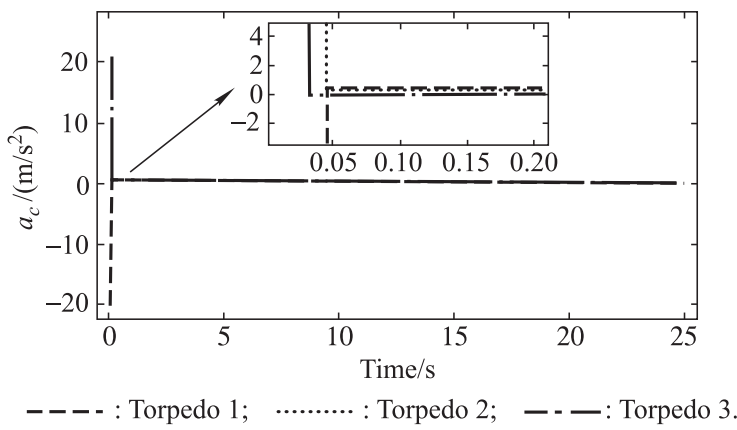

Fig. 11 Guidance law commands $a_{c}$ for different torpedoes with same impact time

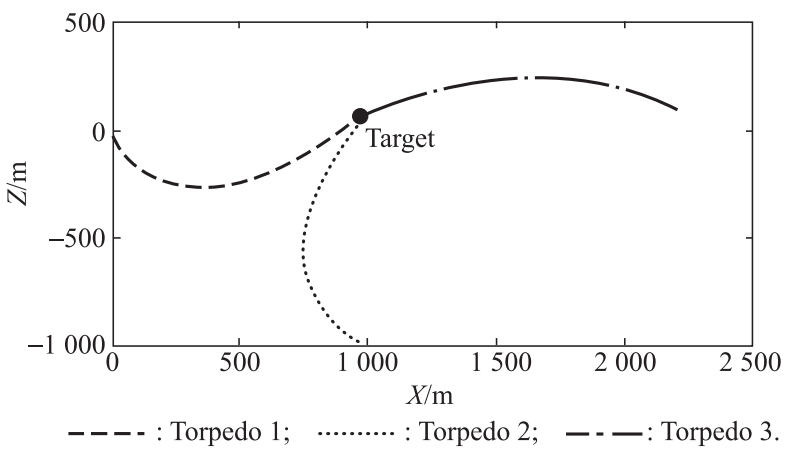

Fig. 12 Trajectories for different torpedoes with same impact time

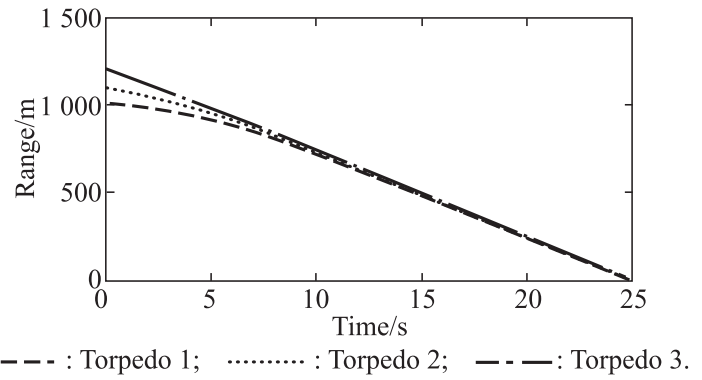

Fig. 13 Ranges for different torpedoes with same impact time

\section{Conclusions}

In this paper, the issue of the guidance system with impact time is discussed, in order to improve the guidance performance, ITCG is proposed based on Lyapunov stability theorem and SMC, then the influence of the singularity problem of guidance command is also analyzed. The proposed ITCG in this paper is compared with the existing ITCG in [2], and simulation results show that ITCG has better performance. The ITCG we present can also be applied for salvo attack. The ITCG can be studied combined with impact angle constraints against maneuvering targets in further related works.

\section{References}

[1] M. Kim, Y. Kim. Lyapunov-based pursuit guidance law with impact angle constraint. IFAC Proceedings Volumes, 2014, 47(3): $2509-2514$.

[2] M. Kim, B. Jung, B. Han, et al. Lyapunov-based impact time control guidance laws against stationary targets. IEEE Trans. on Aerospace and Electronic Systems, 2015, 51(2): $1111-$ 1122.

[3] S. Xiong, W. Wang, X. Liu, et al. Guidance law against maneuvering targets with intercept angle constraint. ISA Transactions, 2014, 53(4): $1332-1342$.

[4] S. R. Kumar, S. Rao, D. Ghose. Non-singular terminal sliding mode guidance and control with terminal angle constraints for non-maneuvering targets. Proc. of the 12th IEEE International Workshop on Variable Structure Systems, 2012: 291 - 296.

[5] B. S. Kim, J. G. Lee, H. S. Han. Biased PNG law for impact with angular constraint. IEEE Trans. on Aerospace and Electronic Systems, 1998, 34(1): $277-288$.

[6] C. H. Lee, T. H. Kim, M. J. Tahk, et al. Polynomial guidance laws considering terminal impact angle and acceleration constraints. IEEE Trans. on Aerospace and Electronic Systems, 2013, 49(1): 74-92.

[7] S. R. Kumar, S. Rao, D. Ghose. Nonsingular terminal sliding mode guidance with impact angle constraints. Journal of Guidance, Control, and Dynamics, 2014, 37(4): 1114-1130.

[8] S. R. Kumar, D. Ghose. Sliding mode control based guidance law with impact time constraints. Proc. of the IEEE American Control Conference, 2013: 5760-5765.

[9] Q. Li, W. Zhang, G. Han, et al. Fuzzy sliding mode control guidance law with terminal impact angle and acceleration constraints. Journal of Systems Engineering and Electronics, 2016, 27(3): $664-679$.

[10] I. S. Jeon, J. I. Lee, M. J. Tahk. Impact-time-control guidance law for anti-ship missiles. IEEE Trans. on Control Systems 
Technology, 2006, 14(2): 260-266.

[11] I. S. Jeon, J. I. Lee, M. J. Tahk. Impact-time-control guidance with generalized proportional navigation based on nonlinear formulation. Journal of Guidance, Control, and Dynamics, 2016: 1887-1892.

[12] W. Shang, J. Guo, S. Tang, et al. Impact time guidance law considering autopilot dynamics based on variable coefficients strategy for maneuvering target. Mathematical Problems in Engineering, 2015: 1-10.

[13] Y. Zhao, Y. Sheng, X. Liu. Sliding mode control based guidance law with impact angle constraint. Chinese Journal of Aeronautics, 2014, 27(1): $145-152$.

[14] Z. Yang, H. Wang, D. Lin, et al. A new impact time and angle control guidance law for stationary and nonmaneuvering targets. International Journal of Aerospace Engineering, 2016: $1-14$.

[15] Y. Zhang, X. Wang, H. Wu. Impact time control guidance law with field of view constraint. Aerospace Science and Technology, 2014, 39: $361-369$.

[16] Y. Zhang, X. Wang, G. Ma. Impact time control guidance law with large impact angle constraint. Proc. of the Institution of Mechanical Engineers, Part G: Journal of Aerospace Engineering, 2015, 229(11): 2119-2131.

[17] S. R. Kumar, D. Ghose. Impact time guidance for large heading errors using sliding mode control. IEEE Trans. on Aerospace and Electronic Systems, 2015, 51(4): 3123 - 3138.

[18] Y. Zhao, Y. Sheng, X. Liu. Analytical impact time and angle guidance via time-varying sliding mode technique. ISA Transactions, 2016, 62: 164-176.

[19] A. Saleem, A. Ratnoo. Lyapunov-based guidance law for impact time control and simultaneous arrival. Journal of Guidance, Control, and Dynamics, 2015, 39(1): 164-173.

[20] S. R. Kumar, D. Ghose. Impact time and angle control guidance. Proc. of AIAA Guidance, Navigation, and Control Conference, 2015: 1-22.

[21] D. Cho, H. J. Kim, M. J. Tahk. Nonsingular sliding mode guidance for impact time control. Journal of Guidance, Control, and Dynamics, 2015, 39(1): $61-68$.

[22] N. Harl, S. N. Balakrishnan. Impact time and angle guidance with sliding mode control. IEEE Trans. on Control Systems Technology, 2012, 20(6): 1436-1449.

[23] Y. Zhao, Y. Sheng, X. Liu. Sliding mode control based guidance law with impact angle constraint. Chinese Journal of Aeronautics, 2014, 27(1): 145-152.

[24] H. Zhou, H. Liu, S. Song. Finite-time sliding mode guidance law design with impact angle constraints. Proc. of the 33rd IEEE Chinese Control Conference, 2014: 675-680.

[25] T. H. Kim, C. H. Lee, I. S. Jeon, et al. Augmented polynomial guidance with impact time and angle constraints. IEEE Trans. on Aerospace and Electronic Systems, 2013, 49(4): $2806-$ 2817.

[26] N. Cho, Y. Kim. Modified pure proportional navigation guidance law for impact time control. Journal of Guidance, Control, and Dynamics, 2016, 39(4): 852-872.

[27] J. Lee. Guidance law for impact time and angle control with control command reshaping. Journal of the Korea Society for Industrial and Applied Mathematics, 2015, 19(3): 271 - 287.

[28] C. K. Ryoo, H. Cho, M. J. Tahk. Time-to-go weighted optimal guidance with impact angle constraints. IEEE Trans. on Control Systems Technology, 2006, 14(3): 483-492.

[29] M. G. Snyder, R. Hull, R. J. Prezenica. A new impact time control guidance law for precise time-on-target missile strike. Proc. of the AIAA Guidance, Navigation, and Control Conference, 2015: 0613-0622.

[30] T. S. No, J. E. Cochran, E. G. Kim. Bank-to-turn guidance law using Lyapunov function and nonzero effort miss. Journal of Guidance, Control, and Dynamics, 2001, 24(2): 255-260.

[31] R. Yanushevsky, W. Boord. Lyapunov approach to guidance laws design. Nonlinear Analysis: Theory, Methods \& Applications, 2005, 63(5): $743-749$.

[32] D. Sang, B. M. Min, M. J. Tahk. Impact angle control guidance law using Lyapunov function and PSO method. SICE Annual Conference, 2007: 2253-2257.

[33] A. Saleem, A. Ratnoo. A nonlinear guidance law for impact time control. Proc. of the American Control Conference, 2015: $651-656$.

[34] Y. B. Shtessel, I. A. Shkolnikov, A. Levant. Guidance and control of missile interceptor using second-order sliding modes. IEEE Trans. on Aerospace and Electronic Systems, 2009, 45(1): $110-124$

\section{Biographies}

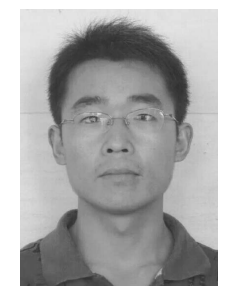

Xiaojian Zhang was born in 1985. He received his B.S. and M.S. degrees from Shaanxi University of Science and Technology in 2009 and 2013 respectively. He is currently working toward his Ph.D. degree in Northwestern Polytechnical University. His research interests are guidance and control of underwater vehicles.

E-mail: xiaojiandr@outlook.com

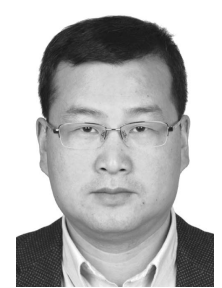

Mingyong Liu was born in 1971. He received his B.S., M.S. and Ph.D. degrees from Northwestern Polytechnical University in 1992, 1995 and 1998 respectively. He is a professor in School of Marine Science and Technology, Northwestern Polytechnical University. His research interests are navigation, guidance and control of underwater vehicles. E-mail: liumingyong@nwpu.edu.cn

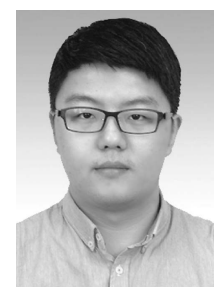

Yang $\mathbf{L i}$ was born in 1987. He received his B.S. and M.S. degrees from Xi' an Shiyou University in 2009 and 2012 respectively. He is currently working toward his Ph.D. degree in Northwestern Polytechnical University. His research interests are navigation and control of underwater super cavitation vehicles. E-mail: liyang_116@yeah.net 